\title{
Pilot randomized phase II trial of the Enhancing Quality of life in Patients (EQUIP) intervention for patients with advanced lung cancer
}

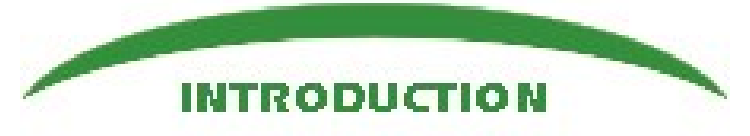

New models of care that are effective and feasible for widespread implementation need to be developed for the delivery of early supportive and palliative care for advanced cancer patients.

The main objective was to pilot the Enhancing Quality of life in Patients (EQUIP) intervention for patients with advanced lung cancer, to determine the feasibility and acceptability of the EQUIP intervention, data completion rate of patient-reported outcome measures in the context of the EQUIP trial and a tentative estimate of efficacy of the EQUIP intervention on quality of life and mood.

\section{MATE RIALS \& MAETHODS}

This was a pilot randomized phase II trial. Patients randomized to the control group received usual care, which comprised standard oncology care as well as referral for palliative care services if deemed appropriate by the primary oncologist. In addition to usual care, patients randomized to the intervention group individually received the EQUIP intervention which comprised four face-to-face educational sessions with a nurse.

\section{RESULTS}

A total of 69 patients were recruited, of which $58(84.1 \%)$ completed all patient assessments. In the intervention group, 30 of 35 patients (85.7\%) completed all four EQUIP sessions. Acceptability of the EQUIP sessions was high: All patients were satisfied with the topics shared and felt they were useful. (Table 1) However, there was no significant difference between intervention and control groups in quality of life at 12 weeks after baseline. (Table 2)

Table 1: Acceptability of EQUIP sessions

\begin{tabular}{lllll}
\hline $\begin{array}{l}\text { Topic of EQUIP } \\
\text { session }\end{array}$ & $\begin{array}{l}\text { Fatigue } \\
(\mathbf{n}=\mathbf{3 4})\end{array}$ & $\begin{array}{l}\text { Breathles } \\
\text { sness } \\
(\mathbf{n}=\mathbf{3 0})\end{array}$ & $\begin{array}{l}\text { Anxiety } \\
(\mathbf{n}=\mathbf{3 3})\end{array}$ & $\begin{array}{l}\text { Recap } \\
(\mathbf{n}=\mathbf{3 0})\end{array}$ \\
$\begin{array}{l}\text { The length of the } \\
\text { session is just } \\
\text { right. }^{\text {a }}\end{array}$ & $33(97.1)$ & $30(100)$ & $32(97.0)$ & $30(100)$ \\
$\begin{array}{l}\text { I feel the topics } \\
\text { shared are } \\
\text { useful. }\end{array}$ & $34(100)$ & $30(100)$ & $33(100)$ & $30(100)$ \\
$\begin{array}{l}\text { I feel satisfied } \\
\text { with the topics }\end{array}$ & $34(100)$ & $30(100)$ & $33(100)$ & $30(100)$ \\
shared. & & & & \\
$\begin{array}{l}\text { I feel comfortable } \\
\text { discussing the } \\
\text { topics. }{ }^{\text {a }}\end{array}$ & $32(94.1)$ & $30(100)$ & $33(100)$ & $30(100)$
\end{tabular}

${ }^{\text {a }}$ Data presented as number (\%) of participants who agreed or strongly agreed with each statement.
Table 2: Quality of life at 12 weeks

\begin{tabular}{|c|c|c|c|c|}
\hline & $\begin{array}{l}\text { Control } \\
(n=30)\end{array}$ & $\begin{array}{l}\text { Intervention } \\
(n=28)\end{array}$ & $\begin{array}{l}\text { Difference after } \\
\text { adjustment for } \\
\text { baseline score }^{b}\end{array}$ & $p$-value ${ }^{b}$ \\
\hline $\begin{array}{l}\text { Physical } \\
\text { wellbeing }\end{array}$ & $\begin{array}{l}22.2 \pm 5.6 \\
{[20.1,24.3]}\end{array}$ & $\begin{array}{l}21.6 \pm 6.8 \\
{[19.0,24.3]}\end{array}$ & $\begin{array}{l}0.1 \pm 1.4 \\
{[-2.6,2.8]}\end{array}$ & 0.92 \\
\hline $\begin{array}{l}\text { Social } \\
\text { wellbeing }\end{array}$ & $\begin{array}{l}23.3 \pm 4.2 \\
{[21.8,24.9]}\end{array}$ & $\begin{array}{l}21.6 \pm 6.6 \\
{[19.1,24.2]}\end{array}$ & $\begin{array}{l}-1.7 \pm 1.4 \\
{[-4.5,1.0]}\end{array}$ & 0.20 \\
\hline $\begin{array}{l}\text { Emotional } \\
\text { wellbeing }\end{array}$ & $\begin{array}{l}19.3 \pm 5.2 \\
{[17.4,21.2]}\end{array}$ & $\begin{array}{l}18.8 \pm 5.2 \\
{[16.8,20.9]}\end{array}$ & $\begin{array}{l}0.4 \pm 1.0 \\
{[-1.5,2.3]}\end{array}$ & 0.71 \\
\hline $\begin{array}{l}\text { Functional } \\
\text { wellbeing }\end{array}$ & $\begin{array}{l}21.0 \pm 5.5 \\
{[19.0,23.1]}\end{array}$ & $\begin{array}{l}18.3 \pm 7.4 \\
{[15.5,21.2]}\end{array}$ & $\begin{array}{l}-2.1 \pm 1.6 \\
{[-5.2,1.0]}\end{array}$ & 0.19 \\
\hline $\begin{array}{l}\text { Overall } \\
\text { quality of life } \\
\text { (FACT-G) }\end{array}$ & $\begin{array}{l}85.9 \pm 14.6 \\
{[80.4,91.3]}\end{array}$ & $\begin{array}{l}80.4 \pm 20.2 \\
{[72.6,88.2]}\end{array}$ & $\begin{array}{l}-3.7 \pm 3.3 \\
{[-10.3,2.9]}\end{array}$ & 0.27 \\
\hline LCS & $\begin{array}{l}21.4 \pm 4.8 \\
{[19.6,23.2]}\end{array}$ & $\begin{array}{l}21.6 \pm 4.7 \\
{[19.7,23.4]}\end{array}$ & $\begin{array}{l}-1.7 \pm 2.6 \\
{[-6.8,3.4]}\end{array}$ & 0.51 \\
\hline Total & $\begin{array}{l}107.2 \pm 18.0 \\
{[100.5,113.9]}\end{array}$ & $\begin{array}{l}102.0 \pm 23.4 \\
{[92.9,111.0]}\end{array}$ & $\begin{array}{l}-5.4 \pm 4.9 \\
{[-15.0,4.2]}\end{array}$ & 0.27 \\
\hline TOI & $\begin{array}{l}64.6 \pm 13.4 \\
{[59.6,69.6]}\end{array}$ & $\begin{array}{l}61.5 \pm 16.0 \\
{[55.3,67.7]}\end{array}$ & $\begin{array}{l}-3.7 \pm 4.0 \\
{[-11.6,4.2]}\end{array}$ & 0.36 \\
\hline
\end{tabular}

${ }^{b}$ Calculated difference in scores and $p$-value obtained from mixed model analysis, with adjustment for baseline score. Presented as mean \pm SE [95\% confidence interval] 